eine Controle für die Formeln ihrer Typen, der Polyglycerinalkohole, zu sein, und bis zu einem gewissen Punkte die durch die Dampfdichten gegebenen zu ersetzen.

\title{
XXXVII. \\ Ueber einige Aether des Glycerins.
}

\author{
Von \\ Reboul und Lourenço. \\ (Compt. rend. t. LII, p. 466.)
}

Wenn man in kleinen Portionen 1 Aeq. Phosphorchlorid zu 1 Aeq. Diäthylglycerin bringt, so tritt eine lebhafte Reaction unter Entwickelung von Chlorwasserstoffsäure ein. Giesst man darauf das entstehende Product nach und nach in eine Lösung von kohlensaurem Kali, um das Phosphoroxychlorür zu zersetzen und die freie Phosphorsäure und Salzsäure zu sättigen, so scheidet sich ein Oel aus von ausserordentlich reizendem Geruch, das schon nach ein-oder zweimaliger Rectification eine bei $184^{\circ}$ siedende Flüssigkeit giebt, deren Analyse zu der Formel führte:

$$
\left.\underset{2\left(\mathrm{G}_{2} \mathrm{H}_{5}\right)}{\mathrm{C}_{3} \mathrm{H}_{5}}\right\} \mathrm{O}_{2}=\mathrm{C}_{7} \mathrm{H}_{15} \boldsymbol{\theta}_{2} \mathrm{Cl} \text {. }
$$

Diese Verbindung ist desshalb der Chlorwasserstoffdiäthyläther des Glycerins, der hierbei nach einer völlig ähnlichen Reaction entsteht, wie die ist bei Einwirkung des Phosphorchlorids auf gewöhnlichen Alkohol.

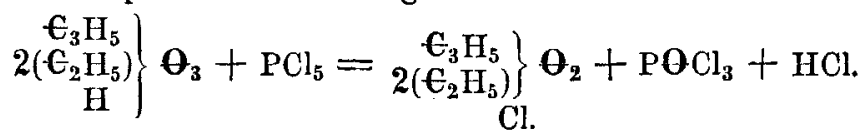

Diese Flüssigkeit ist unlöslich in Wasser, löslich in allen Verhältnissen in Alkohol und in Aether, ihre Dichte ist 1,005 bei $17^{\circ}$; der Dampf derselben reizt Nase und Augen sehr stark. Die Flüssigkeit brennt mit grüngesäumter Flanıme. 
254 Reboul u. Lourenço: Ueber einige Aether des Glycerins.

Die Verbindung wird von concentrirter Aethernatronlösung in der Wäme angegriffen, eine vollständige Umwandlung tritt jedoch erst tin, wenn das Gemisch während einiger Stunden auf $120^{\circ}$ in verschlossenen Iöhren erhitzt wird. Destillirt man alsdann den überschüssigen Alkohol im Wasserbade ab, setzt Wasser zu, um das Chlornatrium $z u$ lösen, und rectificirt die ohenauf schwimmende Flüssigkeit, indem man nur das zwischen 180 und $190^{\circ}$ Uehergehende sammelt, so erhält man eine farblose, klare, ölige Flüssigkeit, unlöslich in Wasser, löslich in Alkohol und Aether, von der Zusammensetzung:

$$
\left.\mathrm{C}_{9} \mathrm{H}_{20} \boldsymbol{\theta}_{3}=\begin{array}{c}
\mathrm{C}_{3} \mathrm{I}_{5} \\
3\left(\mathrm{C}_{2} \mathrm{H}_{5}\right)
\end{array}\right\} \boldsymbol{\Theta}_{3} \text {. }
$$

Diess ist das Triäthylglycerin, das man auch erhält durch Einwirkung des Jodäthyls auf Diäthylglycerinnatron, jedoch in diesem Falle nie frei von Diäthylglycerin, weil man letzteres nicht vollkommen in sein Natronderivat durch Behandlung mit Natrium umwandeln kann. Es ist auch unmöglich, das Diäthylglycerin durch Waschen mit Wasser zu entfernen, in welchem es löslich ist, während das Triäthylglycerin sich darin nicht löst; letzteres löst in diesem Falle das Diäthylglycerin vorzugsweise vor dem Wasser auf.

Eine zweite allgemeine Methode, nach welcher man die Glycerinäther der dritten Reihe erhält, besteht in der directen Vereinigung der Anhydride dieser Aether oder der Aether des Glycids mit den gewöhnlichen Aethern. So entsteht z. B. beim Erhitzen auf $\mathbf{2 0 0}^{\circ}$ von Epichlorhydrin mit Bromäthyl in verschlossenen Gefässen durch directe Vereinigung einer gewissen Menge beider Körper eine schwere Flüssigkeit, die unlöslich in Wasser, löslich in Alkohol und in Aether in allen Verhältnissen ist, bei 186-188 ${ }^{\circ}$ siedet und deren Geruch an den des Chlorwasserstoffdiäthylglycerins erinnert, aber nicht so scharf wie dieser ist. Die Analyse führte zur Formel:

$$
\epsilon_{5} \mathrm{H}_{10} \boldsymbol{\theta C l B r}=\left\{\begin{array}{l}
\epsilon_{3} \mathrm{H}_{5} \\
\epsilon_{2} \mathrm{H}_{5}
\end{array}\right\} \boldsymbol{\theta}
$$

$\mathrm{ClBr}$ 
Die Entstehung dieser Verbindung wird durch die Gleichung ausgedrückt:

$$
\underbrace{\mathrm{C}_{3} \mathrm{H}_{5} \boldsymbol{\theta C l}+}_{\text {Epichlorhydrin. }} \mathrm{G}_{2} \mathrm{H}_{5} \mathrm{Br}=\underbrace{\left\{\begin{array}{l}
\mathrm{C}_{3} \mathrm{H}_{5} \\
\mathrm{C}_{2} \mathrm{H}_{5}
\end{array}\right\} \theta .}_{\text {Aethyl-Chlor- }}
$$

Bromwasserstoff-Glyccrin.

Diese Beispricle genügen, die Bedingungen zu bezeichnen, unter wolchen sich die Glycerinäther der dritten Reihe bilden. Die beschriebenen Verbindungen körnen als Typen dienen, von denen man die Homologen belielig darstellen kann.

Vergleicht man die Siedepunkte der drei Aethyläther des G]ycerins:

$$
\begin{aligned}
& \text { Aethylglycerin } 230^{\circ} \text { - Differenz } 37^{\circ}
\end{aligned}
$$

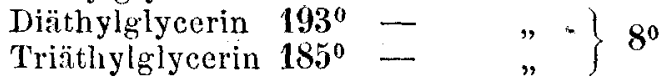

so sieht man, dass durch Substitution des dritten Aequivalentes typischen Wasserstoffes im Glycerin durch Aethyl der Siedepunkt nur wenig geändert wird, er sinkt nur um $8^{0}$, während er durch Substitution des zweiten Aequivalentes um $37^{\circ}$, durch die des ersten Aequivalentes aber um $50^{\circ}$ fällt.

\title{
XXXVIII.
}

\section{Zersetzung der Aether durch wasserfreie Alkalien.}

\author{
Von \\ Berthelot und A. de Fleurieu. \\ (Compt. rend.t. LL, p. 1020.)
}

Man nimmt an, dass bei Zersetzung der Aether durch die Alkalien die Mitwirkung der Elemente des'Wassers erforderlich sei, und es scheint diess ganz begründet zu sein, da die Zerlegung der Aether in Säuren und Alkohol 